\title{
The intermixing and strain effects on electroluminescence of SiGe dots
}

\author{
M. H. Liao \\ Graduate Institute of Electro-Optical Engineering, National Taiwan University, Taipei, \\ Taiwan 106, Republic of China and Department of Electrical Engineering, National Taiwan University, \\ Taipei, Taiwan 106, Republic of China \\ C.-H. Lee and T. A. Hung \\ Graduate Institute of Electronics Engineering, National Taiwan University, Taipei, Taiwan 106, \\ Republic of China and Department of Electrical Engineering, National Taiwan University, Taipei, \\ Taiwan 106, Republic of China \\ C. W. Liu ${ }^{\text {a) }}$ \\ Graduate Institute of Electro-Optical Engineering, National Taiwan University, Taipei, Taiwan 106, \\ Republic of China; Graduate Institute of Electronics Engineering, National Taiwan University, Taipei, \\ Taiwan 106, Republic of China; and Department of Electrical Engineering, National Taiwan University, \\ Taipei, Taiwan 106, Republic of China
}

(Received 1 May 2007; accepted 20 July 2007; published online 14 September 2007)

\begin{abstract}
Secondary-ion mass spectroscopy, energy dispersion spectrometry, and Raman spectroscopy reveal that $\mathrm{SiGe}$ dots grown by ultrahigh-vacuum chemical vapor deposition at $600{ }^{\circ} \mathrm{C}$ exhibit significant intermixing with an average Ge composition of $\sim 50 \%$. Raman spectroscopy shows the top SiGe quantum dots of the 20-layer sample to be more relaxed than those of the 5-layer samples. As a result, the electroluminescence from the top $\mathrm{SiGe}$ quantum dots of the 20-layer sample has the higher peak energy at $\sim 0.84 \mathrm{eV}$ as compared to $\sim 0.82 \mathrm{eV}$ for the 5-layer sample. The external tensile mechanical strain can compensate the built-in compressive strain of SiGe quantum dots and increase electroluminescence energy. (c) 2007 American Institute of Physics.
\end{abstract}

[DOI: $10.1063 / 1.2777686]$

\section{INTRODUCTION}

Recently, the growth of three-dimensional (3D) semiconductor islands and the study of their physical properties have been a very active area of research. ${ }^{1,2}$ The interest in selfassembled Ge-rich dots on $\mathrm{Si}$ is driven by the integration of quantum functional devices with the conventional $\mathrm{Si}$ circuitry. ${ }^{3}$ The accurate control of the size, strain, and composition is crucial for the exploitation of these nanostructures in the quantum devices. The shape of the $\mathrm{Si}_{1-x} \mathrm{Ge}_{x}$ islands, ${ }^{1}$ Ge composition, ${ }^{4}$ and strain distribution ${ }^{5}$ have been investigated. Most of the results show that intermixing and strain relaxation are functions of annealing temperature ${ }^{4,5}$ in the multilayer structures. ${ }^{5}$ Some related electronic and optical properties of SiGe dots due to diffusion and strain have also been reported. ${ }^{6-8}$ In this work, besides the material characterization, the intermixing and strain effects on electroluminescence (EL) from a metal-insulator-semiconductor (MIS) tunneling diode are studied comprehensively. Moreover, external strain can further change the strain state of the $\mathrm{SiGe}$ dots, and can be potentially used for the device design. These results are corroborated by secondary-ion mass spectroscopy (SIMS), energy dispersion spectrometry (EDS), Raman spectroscopy, and cross-sectional transmission electron microscopy (TEM) to provide a consistent picture of composition and strain effects on the optical properties of SiGe dots.

\footnotetext{
${ }^{a)}$ Author to whom correspondence should be addressed. Electronic mail: chee@cc.ee.ntu.tw
}

\section{EXPERIMENT}

The SiGe dots were grown on $p$-type $\mathrm{Si}(001)$ substrates by ultrahigh-vacuum chemical vapor deposition (UHV CVD). After the growth of 100-nm-thick Si buffer, 5 and 20 periods of $\mathrm{Ge} / \mathrm{Si}$ bilayers were grown to form the selfassembled SiGe dots at the temperature of $600{ }^{\circ} \mathrm{C}$ under the Stranski-Krastanov (SK) growth mode. The dot layers with the $\sim 100 \mathrm{~nm}$ base and $\sim 7 \mathrm{~nm}$ height are separated by the $\mathrm{Si}$ spacer. A $3 \mathrm{~nm} \mathrm{Si} \mathrm{cap} \mathrm{layer} \mathrm{was} \mathrm{deposited} \mathrm{above} \mathrm{the} \mathrm{top}$ layer of the self-assembled SiGe quantum dot (QD) layer as a cap layer. Liquid phase deposition (LPD) oxide is subsequently deposited on the $\mathrm{Si}$ cap to act as the tunneling oxide for MIS diodes. The MIS tunneling diode has the top Al gate electrode with the circular area $\left(3.14 \mathrm{~mm}^{2}\right)$ defined by the shadow mask. The other $\mathrm{Al}$ contact with the area of $\sim 1 \mathrm{~cm}^{2}$ is on the back of the wafer as the Ohmic contact. Due to the traps in LPD oxide, the trap-assisted tunneling allows significant current through the oxide and yields electroluminescence.

The $488 \mathrm{~nm}$ line of argon laser was used as the excitation source for the Raman measurement to investigate the Ge composition and strain effect in the SiGe dots. The laser beam was focused on the sample surface with a spot size of $\sim 1 \mu \mathrm{m}$. Backscattering light was collected and recorded by a triple grating spectrometer with a liquid-nitrogen-cooled charge coupled device. The Raman spectra have a resolution of $0.2 \mathrm{~cm}^{-1}$ and the Lorentzian line shape was used to determine the peak position of the line shape. 


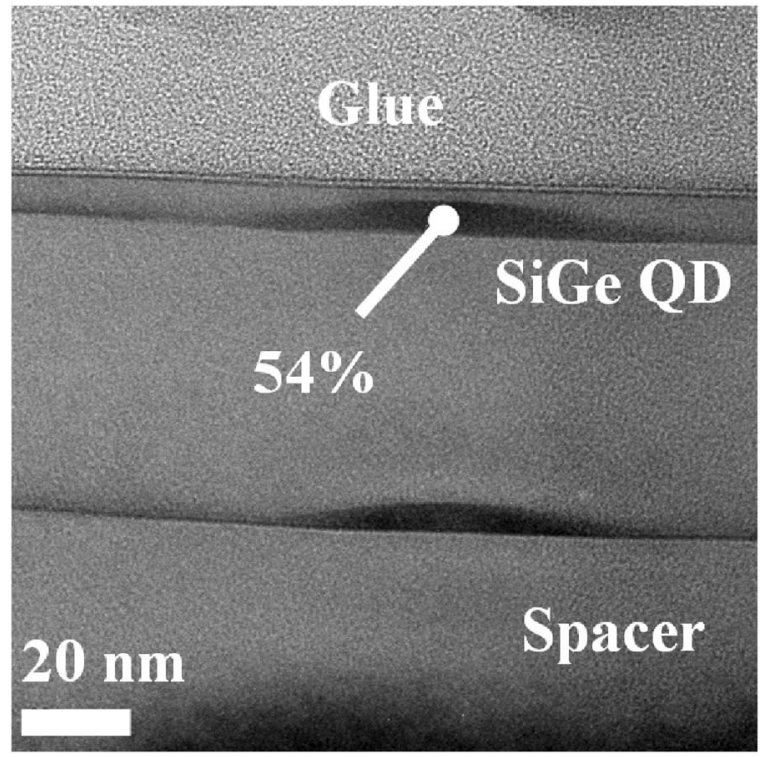

FIG. 1. Cross-sectional TEM micrograph of the 20-layer self-assembled $\mathrm{SiGe}$ quantum dots. The Ge concentration marked in the figure is determined by energy dispersion spectrometry.

For EL measurement, the negative gate voltage drives electrons to tunnel from the gate electrode to the $p$-type semiconductor and these tunneling electrons can recombine with holes in the SiGe dots to have radiative recombination. The details of electroluminescence mechanism can be found in Ref. 5. The MIS tunneling diode was mounted on a mechanical holder inside a liquid-nitrogen cryostat to lower the temperature. Infrared emission from the device was collected with a lens and guided to the input slit of the monochromator. A chopper was placed in front of the monochromator to modulate the light. The lock-in amplifier was synchronized with the frequency of the chopper to filter the noise and to detect the signal. An InGaAs detector placed at the exit of the monochromator was used to detect the signal.

\section{RESULTS AND DISCUSSION}

Figure 1 shows the cross-sectional TEM image with the EDS measurement of the 20-layer SiGe QD structure. Serious intermixing between $\mathrm{Si}$ and $\mathrm{Ge}$ during the thermal growth process is observed. The EDS measurement of the dot marked in Fig. 1 shows that the Ge composition of the SiGe dots at the top layer is about $54 \%$ and the Ge compositions of the middle and bottom dots are $46 \%$ and $40 \%$, respectively. The bottom dots have the highest thermal budget, and thus the lowest Ge composition. Figure 2 shows the SIMS profile of Ge atoms from the 20-layer SiGe QD structure with liquid phase deposited oxynitride on the top ${ }^{9}$ and the EDS measurement on the same sample before the oxide deposition. From the SIMS profiles (Fig. 2), Ge composition in the dot increases with the increasing layer number starting from the substrate due to the less thermal budget after QD formation. Note that the Ge composition in SiGe dots measured by the SIMS profiles is smaller as compared with the EDS measurement due to the limited depth resolution for the thin dots $(7 \mathrm{~nm})$ and the average effect of wetting layers and SiGe dots. The results for SIMS and EDS measurements

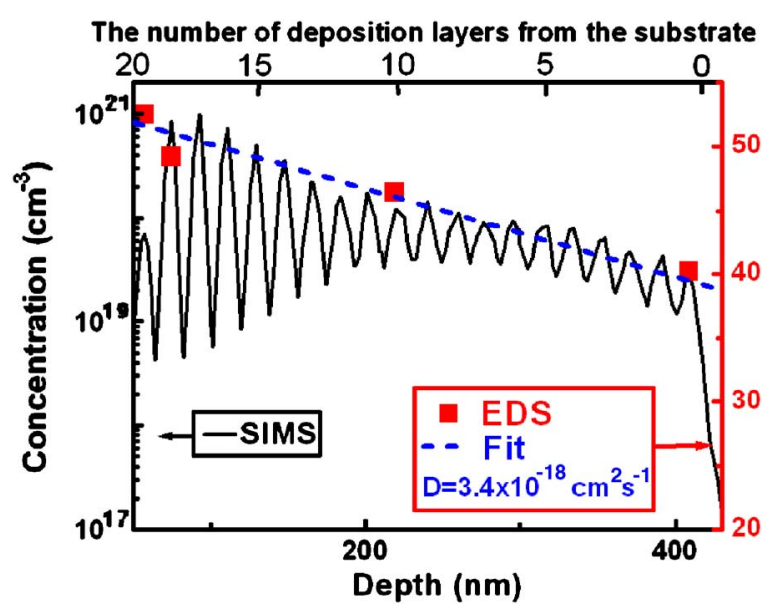

FIG. 2. The secondary-ion mass spectroscopy and energy dispersion spectrometry profiles of Ge atoms. The dashed line is the fitting curve from the one-dimensional interdiffusion model to extract the diffusivity $(D)$ in the SiGe quantum dots.

(Fig. 2) show the same trend except for the top SiGe QD layer. The Ge signal of the top SiGe QD layer in the SIMS profiles is smaller than those of the other dots because the top SiGe QD layer of the 20-layer sample structure is etched by $\mathrm{NH}_{4} \mathrm{OH}$ in the oxynitride process. ${ }^{9}$ Since our SiGe dots have a wide base $(\sim 100 \mathrm{~nm})$ and a short height $(\sim 7 \mathrm{~nm})$, the dots can be considered as quantum wells and the overgrowth when surface can be ignored in the Si/Ge interdiffusion to simplify the quantitative analysis. Thus, we apply a one-dimensional interdiffusion model ${ }^{10,11}$ to analyze our data:

$$
\begin{aligned}
\chi_{\mathrm{Ge}}(z)= & \chi_{\mathrm{Ge}}^{0} / 2\{-\operatorname{erf}[(-W / 2+z) / 2 L] \\
& +\operatorname{erf}[(W / 2+z) / 2 L]\},
\end{aligned}
$$

where $\chi_{\mathrm{Ge}}^{0}$ is the initial Ge concentration, $W$ is the quantum dot thickness, $z$ is the position along the growth direction ( $z=0$ at the center), erf is the error function, $L$ is the interdiffusion length defined as $L=\sqrt{D t}, D$ is the diffusivity of $\mathrm{Si}$ in $\mathrm{Ge}$, and $t$ is the time of thermal growth process. The diffusivity $(D)$ of $3.4 \times 10^{-18} \mathrm{~cm}^{2} \mathrm{~s}^{-1}$ at $600{ }^{\circ} \mathrm{C}$ is extracted from the fitting of the Ge composition in the EDS measurement and is shown in Fig. 3 along with data for the diffusivity of $\mathrm{Si}$ in $\mathrm{Ge}$ from literatures. ${ }^{11-14}$

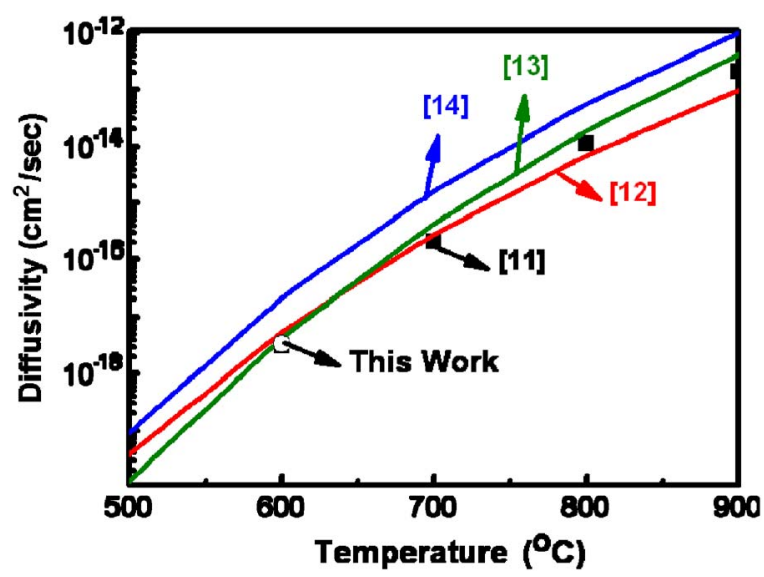

FIG. 3. The diffusion coefficient of $\mathrm{Si}$ in $\mathrm{Ge}$ as a function of temperature 


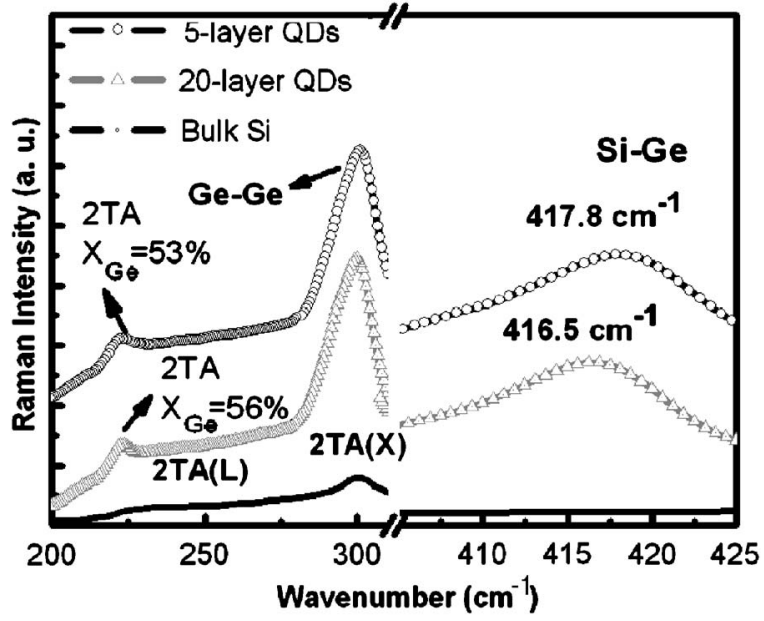

FIG. 4. The Raman spectra of 5-layer SiGe quantum dots, 20-layer SiGe quantum dots, and bulk $\mathrm{Si}$.

Figure 4 shows Raman spectra of the 5-layer SiGe dots, 20-layer SiGe dots, and bulk Si using the $488 \mathrm{~nm}$ laser excitation. Besides the strong $\mathrm{Si}$ signal at $520 \mathrm{~cm}^{-1}$, the $\mathrm{Si}-\mathrm{Ge}$ phonon, Ge-Ge phonon, and $\mathrm{Si}-\mathrm{Ge} 2 \mathrm{TA}$ phonon of $\mathrm{SiGe}$ dots were also observed. The Si-Ge 2TA phonon signal $\left(\sim 220 \mathrm{~cm}^{-1}\right)$ is insensitive to strain and is a strong function of $\mathrm{Ge}$ composition, ${ }^{15,16}$ while the $\mathrm{Si}-\mathrm{Ge}$ phonon peak $\left(\sim 417 \mathrm{~cm}^{-1}\right)$ is sensitive to both the strain and $\mathrm{Ge}$ composition. ${ }^{17}$ Based on the experimental results of the 2TA phonon mode in $\mathrm{Si}_{1-x} \mathrm{Ge}_{x}$ alloys, ${ }^{16}$ the wave number of $2 \mathrm{TA}$ mode at $\sim 220$ and $\sim 222 \mathrm{~cm}^{-1}$ indicates that the average $\mathrm{Ge}$ composition is $\sim 53 \%$ and $\sim 56 \%$ in the top several SiGe QD layers for the 5-layer sample and 20-layer sample, respectively. The signals are distinct from the weak $2 \mathrm{TA}(\mathrm{L})$ phonon mode in the $\mathrm{Si}$ at $225.6 \mathrm{~cm}^{-1}$. The results are reasonable, since the higher compressive strain in the top SiGe dots of the 5-layer sample increases the Ge diffusivity into $\mathrm{Si}$ and leads the lower Ge concentration in the top layer of the 5-layer sample. In this work, the strain difference between the top several $\mathrm{SiGe}$ layers of the 5-layer sample $(\sim 0.94 \%)$ and the 20 -layer sample $(\sim 0.76 \%)$ is $\sim 0.18 \%$, measured by the SiGe phonon peak around $\sim 416 \mathrm{~cm}^{-1}$. Using the parameters in Ref. 18, the Ge diffusivity into $\mathrm{Si}$ is calculated to be 1.7 times of the diffusivity in the unstrained SiGe. Note that due to the absorption depth $(\sim 50 \mathrm{~nm})$ of the $488 \mathrm{~nm}$ argon laser in $\mathrm{Si}_{0.45} \mathrm{Ge}_{0.55}$ (Ref. 19) and exponential decay of absorption from the surface of the sample, the top several SiGe layers of these two samples contribute more significantly in Raman signals than the bottom layers. From the shift of the $\mathrm{Si}-\mathrm{Ge}$ phonon and the theoretical calculation, ${ }^{20,21}$ the top several $\mathrm{SiGe}$ QD layers are $\sim 56 \%$ and $\sim 66 \%$ relaxed in average for the 5-layer sample and the 20-layer sample, respectively. ${ }^{22}$ The corresponding strains are $\sim 0.94 \%$ and $\sim 0.76 \%$ for the 5-layer sample and 20 layer sample, respectively. The accumulation of the strain relaxation for subsequent QD layers may be responsible for the more relaxation of the 20-layer sample.

Figure 5 shows the Raman spectra of the 20-layer SiGe dots with and without external tensile strain. The experimental setup to apply the mechanical biaxial tensile strain is

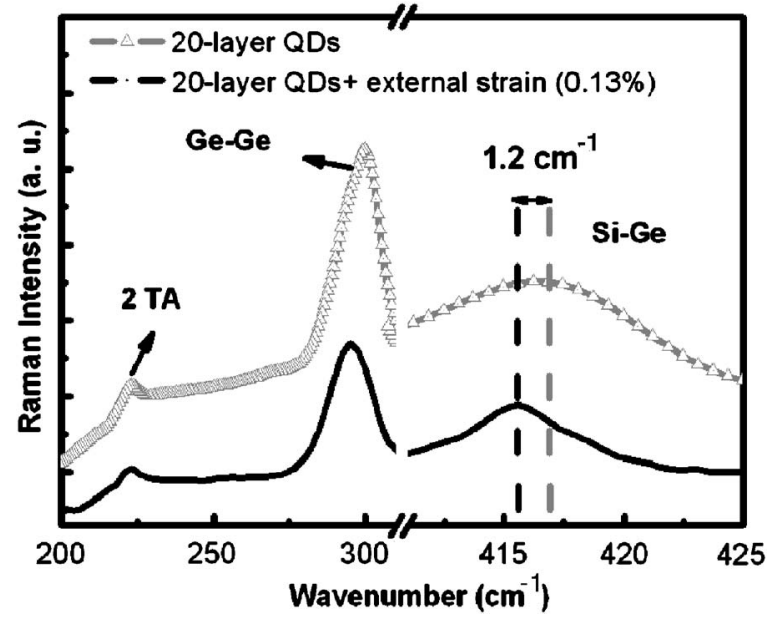

FIG. 5. The Raman spectra of 20-layer SiGe quantum dots with and without external mechanical strain. The shift of Si-Ge peak under the mechanical strain indicates $0.13 \%$ biaxial external tensile strain.

similar to Ref. 23. Under the external biaxial tensile strain, the $\mathrm{Si}-\mathrm{Ge}$ phonon peak in the 20-layer SiGe dots (Fig. 5) shifts toward the relaxed position due to the compensation of the built-in compressive strain by external tensile strain. The Raman shift of $1.2 \mathrm{~cm}^{-1}$ under the external strain indicates the biaxial tensile strain of $0.13 \%$. All the Raman peak positions are extracted by the curve fitting using Lorentzian profiles.

The EL spectra of the SiGe QD MIS tunneling diodes and the recombination mechanism are shown in Figs. 6 and 7 , respectively. At negative bias, the electrons tunnel from the $\mathrm{Al}$ gate to the semiconductor. Meanwhile, the negative gate bias also attracts holes in the accumulation region, and the tunneling electrons can recombine with holes at the oxide/Si interface and in the $\mathrm{SiGe}$ dots to emit $\sim 1.1$ and $\sim 1.5 \mu \mathrm{m}$ infrared, respectively. The MIS light emitting diode (LED) reported in this work operates at the accumulation gate bias (negative gate bias). Most voltage drops at the oxide and thus the electrical field near the oxide/semiconductor

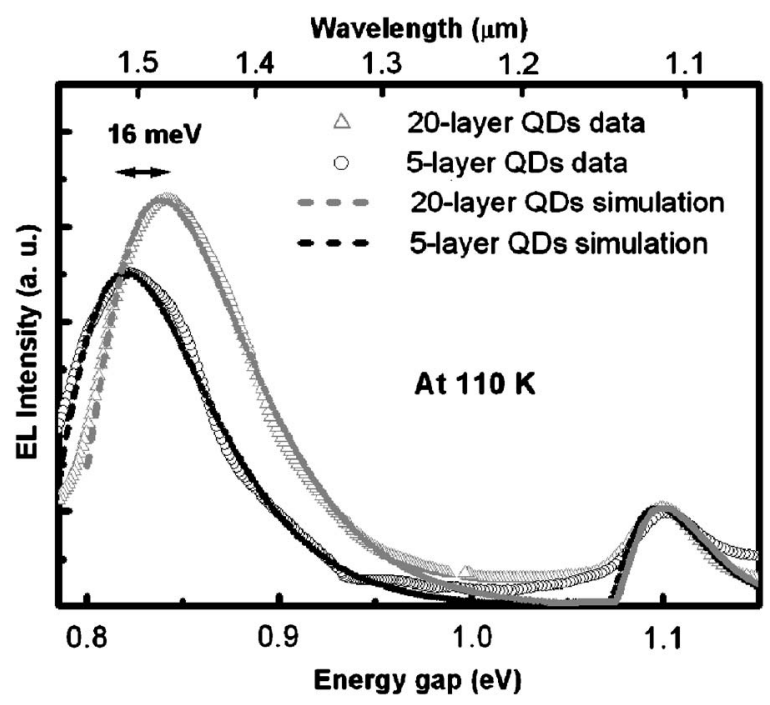

FIG. 6. Electroluminescence spectra of 5-layer sample and 20-layer sample. Due to more relaxation during growth, the 20-layer sample has higher peak energy than the 5-layer sample. 


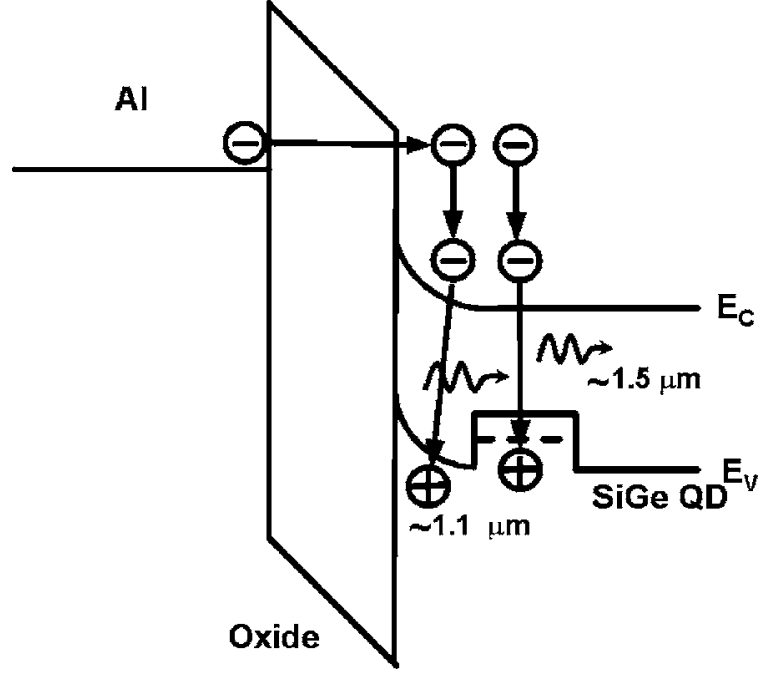

FIG. 7. The energy band diagram for the electroluminescence of the SiGe quantum dot metal-insulator-semiconductor tunneling diode.

surface region are not very strong. The accumulation bias leads to a much higher hole concentration in the top SiGe dots than in the dots far from the surface. Therefore, the EL signal is more likely to be originated from the oxide/ semiconductor interface. Moreover, the smaller area of the top gate electrode $\left(\sim 3.14 \mathrm{~mm}^{2}\right)$ in our device further enhances the current density and radiative probability near the oxide/semiconductor surface region (the current crowding effect). The apparent band gap and device temperature can be obtained by the fitting of the EL spectra with the electronhole plasma recombination model. ${ }^{22}$ The QD peaks at $\sim 0.84$ and $\sim 0.82 \mathrm{eV}$ are extracted for the 20-layer and 5-layer samples, respectively. The emission peak of the 20-layer sample is $16 \mathrm{meV}$ higher than that of the 5-layer sample. It may be due to the smaller compressive strain $(0.76 \%)$ of the top $\mathrm{SiGe}$ dots in the 20-layer sample as compared to the 5-layer sample $(0.94 \%)$. Figure 8 shows the EL spectra at $110 \mathrm{~K}$ for the 20-layer SiGe QD MIS tunneling diodes under external mechanical biaxial tensile strain. After the mechani-

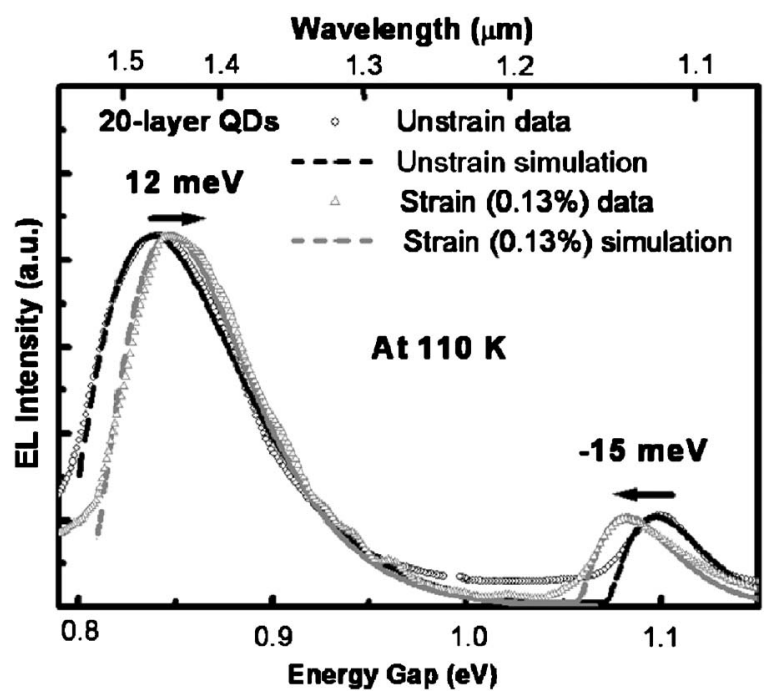

FIG. 8. Electroluminescence spectra at $110 \mathrm{~K}$ of SiGe quantum dots under external mechanical tensile strain $(\sim 0.13 \%)$.

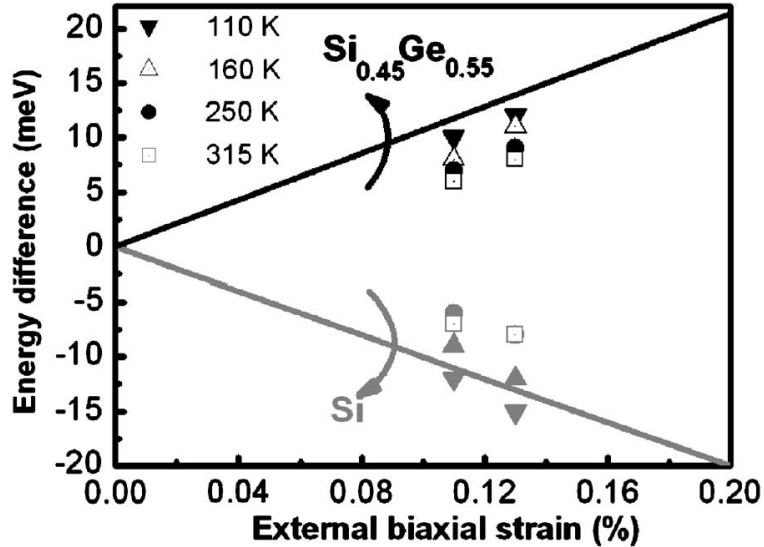

FIG. 9. The theoretical curve and data of the energy gap variation vs strain.

cal tensile strain $(0.13 \%),{ }^{23,24}$ the redshift $(\sim 15 \mathrm{meV})$ of the $\mathrm{Si}$ emission peak at $\sim 1.1 \mu \mathrm{m}$ was observed, while a blueshift $(\sim 12 \mathrm{meV})$ of the SiGe QD peak was observed. The redshift of the Si infrared emission is due to the band gap reduction under external tensile strain. On the other hand, the built-in compressive strain $(0.76 \%)$ of the 20-layer sample is compensated by the external tensile strain $(0.13 \%)$. The decrease of the built-in compressive strain increases the band gap of SiGe dots. The increase of the band gap is responsible for the blueshift of the QD peak. Using the parameters in Ref. 25 along with the formula in Refs. 26 and 27, the theoretical curves and experimental data of $\mathrm{Si}$ and $\mathrm{Si}_{0.45} \mathrm{Ge}_{0.55}$ band gap variations as a function of strain are shown in Fig. 9. The Ge composition of 55\% used in this calculation is the average of Raman and EDS measurement values. The strain in Fig. 9 is measured by Raman shift. The theoretical values of $\Delta E_{g}(\mathrm{Si})$ and $\Delta E_{g}\left(\mathrm{Si}_{0.45} \mathrm{Ge}_{0.55}\right)$ due to strain are calculated to be -100 and $+107 \mathrm{meV} / \%$ strain, respectively, and agree reasonably well with experimental data.

\section{CONCLUSIONS}

We have studied the intermixing and strain in different SiGe QD layer structures by using SIMS, EDS, EL, and Raman measurements. The larger built-in compressive strain $(0.94 \%)$ in the top SiGe dots of the 5-layer sample as compared with the 20-layer sample $(0.76 \%)$ was observed by Raman spectroscopy, and was responsible for the smaller EL energy peak and leads to the larger Si-Ge diffusivity. The external strain induced the EL shift in the opposite direction for the SiGe emission and the Si emission. The EL from a MIS tunneling diode has proven to be an effective technique to probe the optical properties of the SiGe dot near the surface since the radiative recombination occurs near the oxide/ semiconductor interface. The study of intermixing and strain can help future device design based on QD characteristics.

\section{ACKNOWLEDGMENTS}

This work is supported by the National Science Council of ROC under Contract Nos. NSC 95-2221-E-002-357 and NSC 95-2221-E-002-370 and the Applied Materials, Inc. 
${ }^{1}$ J.-M. Baribeau, X. Wu, N. L. Rowell, and D. J. Lockwood, J. Phys.: Condens. Matter 18, R139 (2006).

${ }^{2}$ G. Capellini, M. De Seta, and F. Evangelisti, Appl. Phys. Lett. 78, 303 (2001).

${ }^{3}$ Quantum Semiconductor Devices and Technologies, Electronic Materials Series No. 6 edited by T. P. Pearsall (Wiley, Federal Republic of Germany, 2000).

${ }^{4}$ G. Xia, O. O. Olubuyide, and J. L. Hoyt, Appl. Phys. Lett. 88, 013507 (2006).

${ }^{5}$ M. H. Liao, C.-Y. Yu, T.-H. Guo, C.-H. Lin, and C. W. Liu, IEEE Electron Device Lett. 27, 252 (2006).

${ }^{6}$ X. Z. Liao, J. Zou, D. J. H. Cockayne, J. Wan, Z. M. Liang, G. Jin, and K. L. Wang, Appl. Phys. Lett. 79, 1258 (2001).

${ }^{7}$ M. W. Dashiell, U. Denker, C. Muller, G. Costantini, C. Manzano, K. Kern, and O. G. Schmidt, Appl. Phys. Lett. 80, 1279 (2002).

${ }^{8}$ J. Wan, Y. H. Luo, Z. M. Jiang, G. Jin, J. L. Liu, K. L. Wang, X. Z. Liao, and J. Zou, J. Appl. Phys. 90, 4290 (2001).

${ }^{9}$ P.-S. Kuo, B.-C. Hsu, P.-W. Chen, P. S. Chen, and C. W. Liu, Electrochem. Solid-State Lett. 7, G201 (2004).

${ }^{10}$ P. Boucaud, L. Wu, C. Guedj, F. H. Julien, I. Sajnes, Y. Campidelli, and L. Garchery, J. Appl. Phys. 80, 1414 (1996).

${ }^{11}$ C. W. Liu, Y. D. Tseng, M. Y. Chern, C. L. Chang, and J. C. Strum, J. Appl. Phys.. 85, 2124 (1999).

${ }^{12}$ J. Raisanen, J. Hirvonen, and A. Anttila, Solid-State Electron. 24, 333
(1981).

${ }^{13}$ U. Sodervall and M. Friesel, Defect Diffus. Forum 143, 1053 (1997).

${ }^{14}$ A. Strohm, T. Voss, W. Frank, P. Laitinen, and J. Raisanen, Z. Metallkd. 93, 737 (2002).

${ }^{15}$ P. H. Tan, K. Brunner, D. Bougeard, and G. Abstreiter, Phys. Rev. B 68, 125302 (2003)

${ }^{16}$ J. S. Lannin, Phys. Rev. B 16, 1510 (1977).

${ }^{17}$ M. Stoehr et al., Phys. Rev. B 53, 6923 (1996).

${ }^{18}$ G. Xia, J. L. Hoyt, and M. Canonico, J. Appl. Phys. 101, 044901 (2007).

${ }^{19}$ Silicon Germanium, EMIS Datareviews Series No. 12, edited by E. Kasper (INSPEC, London, 1995), p. 127.

${ }^{20}$ W. J. Brya, Solid State Commun. 12, 253 (1973)

${ }^{21}$ S. D. Gironcoli, Phys. Rev. B 46, 2412 (1992).

${ }^{22}$ C. W. Liu, M. H. Lee, M.-J. Chen, I. C. Lin, and C.-F. Lin, Appl. Phys. Lett. 76, 1516 (2000).

${ }^{23}$ M. H. Liao, S. T. Chang, M. H. Lee, S. Maikap, and C. W. Liu, J. Appl. Phys. 98, 066104 (2005).

${ }^{24}$ M. H. Liao, P.-S. Kuo, S.-R. Jan, S. T. Chang, and C. W. Liu, Appl. Phys. Lett. 88, 143509 (2006).

${ }^{25}$ Properties of Crystalline Silicon, EMIS Datareviews Series No. 20, edited by R. Hull (INSPEC, London, 1999).

${ }^{26}$ C. G. Van de Walle, Phys. Rev. B 34, 5621 (1986).

${ }^{27}$ R. People, Phys. Rev. B 32, 1405 (1985). 